\title{
Crónica sobre el Seminario hispano-alemán de Derecho Público “Después del Tratado de Lisboa"
}

\author{
Antonio $M$ arti del Moral \\ Profesor Titular de Derecho Administrativo \\ Universidad de Jaén
}

Durante los días 4 y 5 de junio de 2008, tuvo lugar en Sevilla un Seminario hispano-alemán de Derecho Público que llevaba por título "Después del Tratado de Lisboa: instituciones, procedimientos de decisión y cuestiones de ejecución", organizado gracias a las excelentes relaciones académicas de los Profesores Stern (Catedrático emérito de Derecho Público de la U niversidad de Colonia), Pérez Moreno (U niversidad de Sevilla), Jiménez-Blanco (Universidad de Jaén) y Pielow (Universidad de Bochum). Fueron unos días intensos de trabajo, aunque también hubo tiempo para el disfrute de la bella ciudad. Participaron un grupo notable de Profesores de Derecho público y administrativo de distintas U niversidades españolas y alemanas, siendo mayoría los que procedían de éstas últimas. En estas páginas intentamos recapitular las principales observaciones que fueron objeto de reflexión durante las ponencias y debates y, por ello, merecen un comentario específico.

Previamente mencionamos el origen de la iniciativa. Este Seminario de Derecho Público fue un episodio más que se añade a una lista de otros anteriores que tuvieron lugar en España y Alemania. En concreto, la idea partió de una sesión de varios Profesores en el Seminario de Derecho Público del Prof. Stern en la Universidad de Colonia en noviembre de 2007, con motivo de la asistencia al Homenaje del difunto Profesor Tettinger, que organizó el Decano de la Facultad de Derecho de la Universidad de Colonia, el Prof. Sachs, en el que se presentó el libro ("Wirtschaft und Gesellschaft im Staat der Gegenwart", (editorial H eymanns, 842 páginas), con contribuciones de varios profesores españoles. Con anterioridad, es fácil comprobar la utilidad de las relaciones académicas entre los Profesores Stern, Tettinger y Jiménez-Blanco que se han materializado en seminarios y publicaciones sobre cuestiones de actualidad, a modo de ejemplo así aconteció con el Comentario a la Carta Europea de los Derechos Fundamentales publicado en 2006 en alemán. 
El programa de las Jornadas se pudo seguir simultáneamente en alemán y castellano gracias a un servicio eficaz de traducción, en un lugar especialmente adaptado en el Foro de Derecho Administrativo dirigido por el Prof. Pérez Moreno. El seminario se agrupó en torno a siete temas, cada uno de ellos estructurado en dos ponencias, una presentada por un profesor alemán y otra por su homónimo español, y su debate posterior, a saber:

- La evolución de la Carta europea de los Derechos Fundamentales (Stern, Universidad de Colonia y Blanke, U niversidad de Erfurt).

- Las instituciones comunitarias (Montoya, U niversidad de Sevilla y Jiménez-Blanco, Universidad de Jaén)

- El principio de subsidiariedad y los mecanismos de control (Pieper, Universidad de Münster y Marti del Moral, Universidad de Jaén)

- Nuevas competencias de la Unión (Pérez Moreno, Pérez Andrés y Sánchez Sáez, Universidad de Sevilla)

- Los servicios de interés económico general (De la Torre Martínez, Universidad de Jaén y Pielow, Universidad de Bochum)

- La adaptación de la Directiva Servicios ("Bolkenstein") de 2006 en España y en Alemania (Cremer, U niversidad de Bochum y Galán Vioque, Universidad de Sevilla)

- $\quad$ Diez años de euro (Geerlings, Universidad de Colonia).

En adelante, comentaremos brevemente el contenido de las ponencias y los debates posteriores sin ánimo de ser exhaustivos, lo que por otra parte, resultaría pretencioso.

La primera ponencia correspondió al Prof. Stern, bien conocido especialmente por el Tratado de 7 tomos "Das Staatsrecht der Bundesrepublik Deutschland" (el último tomo IV-1 es relativo a los derechos fundamentales del individuo, publicado en 2006 y cuenta con más de 2422 páginas; vid. www.klausstern.net), así como por sus trabajos en materia de Derecho comunitario, como otros profesores alemanes (P. H äberle, H.P. I psen, T. O ppermann), quienes persiguen iluminar el proceso de creación de un Derecho constitucional europeo imbricado con el Derecho constitucional de los Estados europeos.

En su intervención el Prof. Stern aludió a los motivos que dieron lugar al nuevo Tratado de Lisboa analizando los últimos desarrollos del proceso de constitucionalización de la Unión Europea. Se comentó la situación paradójica a que ha llevado el Consejo Europeo de Bruselas de 21 y 22 de junio de 2007 y el Proyecto de Tratado de Reforma que se ha elaborado a partir del 
mismo: por un lado, se ha incorporado sustancialmente el contenido del Tratado Constitucional, mientras que, por otro, se ha eliminado el término "Constitución". Se ha pasado así de una Constitución con forma de Tratado (esto es, de un Tratado con contenido constitucional) a una Constitución con nombre de Tratado.

En el debate se analizó el alcance de las modificaciones que se han introducido en relación con el Proyecto de Tratado Constitucional y no se pudo soslayar la voluntad expresa de que no se considere "Constitución" los nuevos textos fundamentales resultantes del Tratado de Lisboa. Ciertamente, la reforma de Lisboa no ha querido proporcionar una base constitucional sólida para pasar de una Constitución material a una Constitución formal europea. Y ello porque no es pacífica la aceptación de esta idea, como se ha demostrado en los resultados negativos de los referéndums a los que se ha sometido el Proyecto de Tratado Constitucional en Francia y Holanda (y recientemente en Irlanda respecto al Tratado de Lisboa, como se verá posteriormente).

EI Prof. Stern en la conferencia inaugural explicó el retorno al método tradicional de revisión de los Tratados, mediante la adopción de un nuevo texto que introduce modificaciones en los Tratados actuales, los cuales seguirán en vigor, lo que supone la dualidad de los Tratados. Así un primer Tratado, el Tratado de reforma que modificará el Tratado de la Unión Europea (TUE) y el Tratado constitutivo de la Comunidad Europea (TCE), que pasará a llamarse "Tratado sobre el Funcionamiento de la U nión Europea", (TFUE). Si bien con matices, puede afirmarse que el primero tendrá un marcado carácter constitucional en el que se expresan los principios y valores (especial mente en los arts. 1 a 7), y el otro más bien una naturaleza típicamente administrativa, por cuanto organiza el funcionamiento de la U nión y determina los ámbitos, la delimitación y las condiciones de ejercicio de sus competencias (art. 1 TFUE).

Pese a la intención de eliminar las referencias constitucionales en los Tratados, no parece que pueda ser admitida esta afirmación en una dimensión material, como puede inferirse de la jurisprudencia del Tribunal de Justicia de la UE. Y ello porque, aunque carezca de una Constitución en sentido formal, la U nión Europea constituye un ordenamiento constitucional autónomo, por mucho que esté estrechamente ligado a los Derechos constitucionales de sus Estados miembros. Además, algunos preceptos del Tratado de la Unión Europea permiten sostener la idea de fondo de una constitucionalización de la Comunidad. En el Preámbulo del Tratado de la CEE y ahora en el artículo 1 TUE de Lisboa hay que notar la referencia a "una unión cada vez más estrecha entre los pueblos de Europa", así como en lo dispuesto en los artículos 
2 ( valores comunes de la UE) y 3 ( bienestar, paz, espacio de libertad y mercado interior) TUE.

También quedó manifiesto que el proceso de la integración europea desde los inicios hasta el Tratado de Lisboa, ha supuesto un avance continuado hacia la constitucionalización de esa U nión de Estados europeos, que se puede denominar como una "evolución constitucional", porque los ideales clásicos de una Constitución han sido aportadosa la U nión en mayor o menor medida por casi todos los Estados miembros (art. 2 TUE). De este modo, Stern entiende que la Unión es una "Comunidad de Derecho constitucional". Ahora bien, también se puso de relieve que la U nión Europea constituye una absoluta novedad institucional: una novedad no reconducible a ninguno de los modelos clásicos de tipo confederal y federal. Ahora bien, todos los asistentes coincidían en afirmar que el Tratado de Lisboa representa una mejora importante de los Tratados existentes, aunque no tenga el carácter constitucional manifiesto que fue la nota predominante del fracasado Proyecto de Tratado Constitucional.

En la siguiente ponencia, el Prof. H.J. Blanke (Universidad de Erfurt) hizo una exposición completa y minuciosa sobre la protección de los derechos fundamentales en la U nión Europea desde la perspectiva del derecho alemán y comunitario europeo. Fue objeto de comentario la famosa jurisprudencia del Tribunal Constitucional Federal alemán desde la primera Sentencia "Solange" en relación con el estándar de protección de los derechos en la Unión Europea. En cuanto a las novedades que introduce el Tratado de Lisboa, el reconocimiento de la fuerza vinculante de la Carta de los Derechos Fundamentales adoptada en el nuevo art. 6 supone un paso adelante en la protección jurídica de los ciudadanos. Las instituciones europeas y los Estados miembros han de respetar este precepto, sin embargo la aceptación de las excepciones británica y polaca en el Protocolo núm. 30 supone una concesión al menos criticable.

Por otro lado, se comentó la importancia de la adhesión de la U nión al Convenio Europeo para la Protección de los Derechos H umanos y de las Libertades Fundamentales, que no modificará las competencias de la U nión que se definen en los Tratados (art. 6.2 TUE), así como el art. 6.3 TUE que prescribe que los derechos fundamentales del Convenio "y los que son fruto de las tradiciones constitucionales comunes a los Estados miembros formarán parte del Derecho de la Unión como principios generales". Todo ello permite una garantía más eficaz en la protección de los derechos.

En una ponencia posterior, la Prof. Encarnación Montoya (Universidad de Sevilla) abordó la estructura y composición de las instituciones comunita- 
rias aludiendo a las novedades que presenta el Tratado de Lisboa. Entre ellas, se consideró la nueva lista de instituciones comunitarias del art. 13 TUE, la formalización del Consejo Europeo como institución y su presidencia estable (art. 15 TUE), así como el papel otorgado en el art. 18 TUE al Alto Representante para Asuntos Exteriores y la Política de Seguridad, quien presidirá el Consejo de Asuntos Exteriores y hablará en nombre de la U nión en aquellos temas en los que exista una posición común, garantizando de este modo una mayor coherencia en la acción exterior de la Unión.

Este órgano se incrusta por primera vez en el seno mismo de la Comisión como Vicepresidente, su nombramiento depende del Consejo Europeo y debe someterse al mismo procedimiento de investidura ante el Parlamento Europeo al que se someten los demás Comisarios. Precisamente sobre esta cuestión, en el debate hubo varias intervenciones interesantes, entre otras, la del Prof. Albert Weber (Catedrático de Derecho Público de la U niversidad de Osnabrück), y en ellas se observó que la posición jurídica del Alto Representante de la U nión, antiguo "Ministro de Asuntos Exteriores de la U nión" según el Proyecto de Tratado Constitucional, puede entrar en colisión con otras instituciones como el Presidente de la Comisión Europea.

En cuanto a la Comisión ahora regulada en el art. 17 TUE, la pérdida del nombramiento de un Comisario por país, como establece el Tratado de Niza, es una importante novedad que se incorporó al Tratado de Lisboa, no sin fuertes resistencias de algunos Estados. A partir de 2014 se reducirá el número de miembros de la Comisión a dos tercios del numero de Estados miembros, lo que aumentará la capacidad de acción de la Comisión y pondrá de manifiesto que los miembros de la Comisión representan los intereses de Europa y no los de sus respectivos países de origen, al tiempo que un sistema de rotación seguirá garantizando la igualdad de participación de todos los Estados miembros (art. 17.5 TUE).

El nuevo Tratado persigue una mayor responsabilidad democrática, con lo que los ciudadanos dispondrán de un mayor control sobre la actuación de la Unión Europea, lo que se revela en distintos asuntos, por ejemplo, la elección por el Parlamento del Presidente de la Comisión, a propuesta del Consejo Europeo (art. 17.7 TUE), establece un claro vínculo con los resultados de las elecciones europeas; se refuerza el control democrático sobre la competencia legislativa delegada en la Comisión mediante un nuevo sistema de supervisión; el procedimiento de revisión, etcétera.

El Prof. Jiménez-Blanco disertó sobre los procedimientos de toma de decisiones de las instituciones comunitarias, aludiendo a que el Tratado de Lis- 
boa pretende "salvar los tesoros de un barco hundido". De partida, el ponente subrayó el propósito del Tratado de huir de toda referencia estatal, lo que explica el abandono de las denominaciones "ley" y "ley marco" que se utilizaron en el Proyecto de Tratado Constitucional y el mantenimiento de las de "reglamento", "directiva" y "decisión" actuales conforme al Tratado de Niza.

De otra parte, mencionó la doble fuente de legitimación de la U nión (Estados y población) en los procedimientos de toma de decisiones por la Comisión (art. 17 TUE) y el Consejo (art. 16 TUE), explicando las nuevas reglas establecidas en el Tratado. De todo ello, deriva un notable incremento del número de sectores en los que los Gobiernos reunidos en el Consejo decidirán por mayoría cualificada en lugar de por unanimidad, con lo que la U nión de veintisiete Estados miembros podrá funcionar en más ámbitos sin verse bloqueada por vetos.

En relación con el Parlamento Europeo, comentó el aumento de los ámbitos materiales que se someterán a colegislación con el Consejo, lo que lleva a la confirmación del Parlamento como colegislador ordinario. Destacó el papel que se otorga al Parlamento Europeo (art. 14 TUE), que pretende alcanzar la "mayoría de edad" que muestran los Parlamentos nacionales, sin tener en cuenta, de otro lado, la crisis actual de muchos de ellos que se caracteriza por la hegemonía del poder ejecutivo.

Con carácter general, también se ponderó el alcance de la democracia en relación con la UE, mencionada como uno de los valores comunes de la UE en el art. 2 TUE, considerando si la UE fuera una democracia directa, pero no lo es, o sólo en la pequeña parte de legitimidad que se transmite a través de las elecciones directas al Parlamento Europeo. La UE sigue siendo principalmente una democracia indirecta, por lo que cada Estado miembro democrático tiene que tomar su propia decisión de acuerdo con su ordenamiento interno.

El Prof. Stefan Pieper (U niversidad de Münster y Director de la Asesoría Jurídica de la O ficina del Presidente de la República en Berlín), realizó una exposición completa sobre el principio de subsidiariedad y el control por los Parlamentos nacionales, mencionando el origen del principio y su desarrollo también en el derecho alemán, materia a la que dedicó una monografía ( "Subsidiarität. Ein Beitrag zur Begrenzung der Gemeinschaftskompetenzen", Ed. Heymann, 1994, 337 páginas). En este sentido, indicó los mecanismos que prescribe el artículo 23 de la Ley Fundamental de Bonn en los apartados 1 a 7, la referencia expresa al principio de subsidiariedad y el principio general de que en los asuntos vinculados a la UE participan el "Bundestag" y los "Länder" 
a través del "Bundesrat" o Senado, lo que demuestra una solución constitucional a la participación de los distintos Estados federados en el proceso de integración europea. También explicó pormenorizadamente la Ley alemana de 2005 que regula esta materia. En relación con el Tratado de Lisboa, explicó el sometimiento de las instituciones comunitarias a los principios de subsidiariedad y proporcionalidad reconocidos en el art. 5 TUE, que no son principios nuevos, al contrario fueron introducidos en el Tratado de Maastricht y en el Protocolo anexo al Tratado de Amsterdam de 1997. Sin embargo, es novedosa la referencia a los niveles regional y local como parámetros para la subsidiariedad en el art. 5 TUE.

A este respecto, la mayor novedad está constituida por el papel que la nueva regulación reconoce a los Parlamentos nacionales. En este sentido, el Prof. Marti (Universidad de Jaén) explicó los mecanismos de control que establece el Tratado de Lisboa para el control del principio de subsidiariedad por los Parlamentos nacionales según lo dispuesto en el art. 12 TUE y en los Protocolos núm. 1 y 2 anejos, centrando su intervención en el sistema de "alerta temprana", que había sido creado en el Proyecto de Tratado Constitucional. Este sistema ha sido reforzado en el Tratado de Lisboa con la ampliación de los plazos a favor de los Parlamentos nacionales frente a la Comisión y la posibilidad de una suerte de veto a sus propuestas, mediante la posibilidad de sacar, lo que se conoce en el argot comunitario, "tarjeta naranja", una solución intermedia entre la "amarilla" (deber de motivación de la propuesta legislativa y nueva toma de consideración) y la "roja" (potestad de paralizar totalmente una propuesta de acto legislativo por los Parlamentos nacionales).

En resumen, el nuevo Tratado de Lisboa convierte a los Parlamentos nacionales, en guardianes del principio de subsidiariedad en un escenario nuevo en el que se permite incluso la interposición del recurso por violación del principio ante el Tribunal de Justicia de la UE, sistema cuya viabilidad fue discutida en el debate posterior.

Los Prof. Pérez Moreno, Pérez Andres y Sánchez Saéz disertaron sobre los nuevos ámbitos políticos de la Unión, observando que el Tratado de Lisboa muestra un mayor énfasis en políticas que benefician visiblemente a los ciudadanos ( nivel de empleo, protección social adecuada, desarrollo sostenible y protección del medio ambiente, incluidos la lucha contra el cambio climático, la energía, el espacio, la investigación, el turismo, el deporte, la salud pública), y que aparecen definidas con claridad. En efecto, el nuevo Tratado otorga más posibilidades de llegar a soluciones flexibles cuando no todos los Estados miembros quieran o puedan seguir adelante al mismo tiempo con determinadas políticas. 
Ahora bien, el principio de atribución reconocida expresamente en el Tratado de Lisboa (art. 5.1 y 2 TUE) implica que la UE no pueda ostentar competencias generales que no le han sido transferidas por los Estados miembros. Así pues, el Tratado de Lisboa mejora la delimitación de las competencias de la Unión con respecto a los Estados miembros, en virtud del principio por el que toda competencia no atribuida a la Unión en los Tratados corresponde a los Estados miembros (art. 4.1 TUE). EI Tratado de Lisboa confirma la especificidad de la organización institucional de la Unión, a la vez que aporta suficientes garantías de que la Unión no se convertirá en un "super-Estado" centralizado y todopoderoso: obligación de respetar la identidad nacional de los Estados miembros (art. 4.2 TUE), sometimiento a los principios de atribución de competencias, subsidiariedad y proporcionalidad (art. 5 TUE); participación de los propios Estados miembros en la toma de decisiones de la U nión; y derecho de cada Estado miembro a retirarse de la U nión (art. 50 TUE).

El Prof. Pérez Moreno inició el examen de las competencias exclusivas (art. 3 TFUE), compartidas (art. 4 TFUE) y complementarias (art. 6 TFUE) de la UE, incidiendo en las dificultades que pueden aparecer para conocer el alcance exacto de cada una de ellas en relación con las competencias de los Estados. En todo caso, las apreciaciones efectuadas ayudaron a entender la distribución de las competencias entre la UE y sus Estados miembros en los nuevos ámbitos políticos de la U nión.

La Prof. Lourdes de la Torre (U niversidad de Jaén) inició la ponencia sobre los servicios de interés económico general explicando los preceptos del Tratado de Lisboa que afectan a su regulación, entre los que resulta de especial interés el art. 14 TFUE. Este precepto modifica el art. 16 vigente $y$, por primera vez en un tratado comunitario, otorga al Parlamento Europeo y al Consejo la potestad de dictar reglamentos que establezcan los principios y condiciones que permitan a los servicios de interés económico general cumplir su cometido, con arreglo al procedimiento legislativo ordinario, "sin perjuicio de la competencia que incumbe a los Estados miembros, dentro del respeto a los Tratados, para prestar, encargar y financiar dichos servicios", precepto cuya aplicación no dejará indiferente a ninguno de los Estados miembros contrarios a una regulación con carácter general de los servicios mencionados.

EI Prof. Pielow (Universidad de Bochum) disertó sobre los servicios de interés económico general, a los que ha dedicado una monografía completa ( "Grundstrukturen öffentlicher Versorgung - Vorgaben des Europäischen Gemein-schaftsrechts sowie des französischen und des deutschen Rechts unter besonderer Berücksichtigung der Elektrizitätswirtschaft", Tübingen, Editorial 
Mohr-Siebeck, 2001, 800 páginas), comentando la dificultad en determinar el concepto de servicios de interés económico general en la jurisprudencia comunitaria así como las dudas en la aplicación de las normas sobre competencia. Como novedad, fue considerado el art. 1 del Protocolo núm. 26 anejo al Tratado de L isboa que reconoce "la amplia capacidad de discreción de las autoridades nacionales, regionales y locales para prestar, encargar y organizar los SIEG, así como el artículo 2 del Protocolo cuando dispone que "Ios Tratados no afectarán en modo alguno a la competencia de los Estados miembros para prestar, encargar y organizar los servicios de interés general que no tengan naturaleza económica".

Las siguientes ponencias correspondieron al Prof. Cremer (Universidad de Bochum) y al Prof. Galán (U niversidad de Sevilla) sobre la adaptación de la Directiva de servicios en el mercado interior (Bolkenstein) de 2006 en España y Alemania, que muestran un panorama bien diferente derivado de la intervención de las Comunidades Autónomas y de las potestades de las Cámaras de Comercio. En la última ponencia titulada "Diez años de la Zona Euro", el Prof. Jörg Geerlings (U niversidad de Colonia), conectó las consecuencias derivadas de la situación económica y financiera en la Unión Europea con las previsiones de los Tratados.

Por último, no se puede obviar las dificultades que recientemente han surgido en el proceso de ratificación del Tratado de Lisboa, tras el 'shock' del rechazo irlandés en el referéndum celebrado el día 12 de junio de 2008. Con todo, el Consejo Europeo celebrado los días 19 y 20 de junio en Bruselas, decidió seguir adelante con el proceso de ratificación hasta el próximo Consejo Europeo de 15 de octubre de 2008.

En la clausura del Seminario, el Prof. Pérez Moreno animó a continuar este tipo de encuentros, que podrían ser el germen de una futura Asociación hispano-alemana de Profesores de Derecho Público, lo que sucedió hace unos años en Sevilla respecto a la actual Asociación hispano-italiana de Profesores de Derecho Administrativo, que cuenta ya con una larga tradición. También el Prof. Pielow y otros profesores alemanes manifestaron su agradecimiento por la buena organización del evento y el trato excelente prestado por los profesores españoles, pero es que además no se dejó pasar la ocasión y se aceptó la idea de celebrar un próximo encuentro en fechas cercanas en Alemania. 